\title{
The origins of life and the mechanisms of biological evolution
}

\author{
Francisco Carrapiço* \\ Universidade de Lisboa, Faculdade de Ciências, Departamento de Biologia Vegetal, Centro de \\ Biologia Ambiental, Bloco C2, Campo Grande, 1749-016 Lisboa, Portugal
}

\begin{abstract}
Evolution is usually taught as the result of mutations and genetic recombinations combined with natural selection, but most living forms have symbiotic relationships with microorganisms, and in this sense symbiogenesis seems to play a very important role in the origin and life evolution. Symbiosis is an important support for the acquisition of new genomes and new metabolic capacities, which drives living forms' evolution. In this sense, the evolutionary changes can be explained by an integrated cooperation between organisms, in which symbiosis acts, not as an exception, but rather as the rule in nature. Beginning with the eukaryotic cell formation, symbiogenesis appears to be the main evolutionary mechanism in the establishment and maintenance of biomes, as well as the foundation of biodiversity, based on rather suddenly evolutionary novelty, which challenges the Darwinian gradualism. These principles can be applied to the life on Earth and beyond.
\end{abstract}

Keywords: biological evolution, origins of life, symbiosis, symbiome

\section{INTRODUCTION}

In a recent work ${ }^{1}$ Gilbert V. Levin has theorized, in a brillant way, the application of Darwinian principles to the evolution of life on Mars, suggesting that the "Mars life evolved from microorganisms identical, or similar to, the early forms that have been identified on Earth" and "Martian and Earth life forms are of common ancestry, therefore genetically similar, and sharing common metabolism and chirality". We believe that these principles can be extended, incorporating symbiogenic mechanisms in biological evolution.

For many scientists, symbiosis is still considered as an exception among biological phenomena and not as a common rule in nature. This issue reflects itself in an interpretative reality of the biological evolution where symbiosis remains mainly peripherical to contemporary evolutionary theory ${ }^{2}$, and often considered as a special type of host-parasite relationship. The approach of the traditional evolutionist authors, in relation to this phenomenon, consists in a point of view that emphatizes symbiosis as a residual aspect of the evolution problem. Recent data ${ }^{3,4,5}$, however, point to the exact opposite direction, demonstrating that symbiosis is a factor of evolutive change, giving rise rather suddenly to evolutionary novelty which, in our point of view, challenges several Darwinian and neo-Darwinian tenets, namely gradualism. This situation has important consequences, not only in the way we perceive evolution, but also in the conceptual organization and tenets of Modern Synthesis.

\section{SYMBIOGENESIS AND THE ORIGIN AND EVOLUTION OF LIFE}

After the first studies on lichens in the XIX century, namely the knowledge of its biological structure and the symbiotic relationships established between the alga and the fungi, symbiosis appears as a new mode of organization of living

* e-mail: f.carrapico@fc.ul.pt; phone: +351 217500381; fax: + 351 217500048; web: azolla.fc.ul.pt

Instruments, Methods, and Missions for Astrobiology IX, edited by

Richard B. Hoover, Gilbert V. Levin, Alexei Yu. Rozanov, Alexander Spirin,

Proc. of SPIE Vol. 6309, 63090O, (2006) $\cdot 0277-786$ X/06/\$15 $\cdot$ doi: 10.1117/12.681946

Proc. of SPIE Vol. 6309 630900-1 
beings and a new way to produce novelty. In 1867, Simon Schwendener proposes the "dual hypothesis", in the Swiss Natural History Society annual meeting held in Rheinfelden to explain the nature of lichens, indicating that those are an association of two organisms, a fungus and an alga, behaving as "master-slave" relationship". Eleven years later, Heinrich Anton de Bary introduces the concept of symbiosis (from Greek, meaning "living together") as "the living together of unlike named organisms" in a communication entitled "Ueber Symbiose" (On Symbiosis) during a meeting at Cassel (Germany) of the Congress of German Naturalists and Physicians. De Bary used this term while discussing the presence of the cyanobacteria in the leaf cavity of Azolla, as well as the nature of lichens and the role of the alga and fungi in this association ${ }^{8}$. These two concepts are important to understand the next theoretical formulation symbiogenesis - postulated by Constantin Merezhkowsky in his book "The Theory of two Plasms as Foundation of Symbiogenesis, New Doctrine on the Origin of Organisms" written in Russian and published in 1909. Merezhkowsky wrote this work during his stay at the Kazan University, defining the concept of symbiogenesis as "the origin of organisms by the combination or by the association of two or several beings which enter into symbiosis". In his work he introduces not only the new concepts on the symbiogenesis field, but he also develops some important ideas about the origin of life, namely related with the role of extremophiles in that scenario ${ }^{9,10}$. The symbiogenesis concept was a landmark for the development of further studies on biology and evolution, even it was not well understood at the time it was formulated or received proper attention from the scientific community.

In a simple way, symbiogenesis should be understood as an evolutive mechanism and symbiosis as the vehicle, through which that mechanism unfolds ${ }^{10}$. This fact represents a point of view which is opposed to that of the neo-Darwinism or Modern Synthesis theory. Thus, according to today's dominant theory, evolution is a gradual process essentially consisting of a natural selection conducted on minimal phenotypical variations. However, most living forms have symbiotic relationships with microorganisms, and, in that sense, symbiosis seems to play a very important role in the origin, organization and life evolution. It is an important support for the acquisition of new genomes and new metabolic capacities, which drives living forms' organization and evolution. In this sense, the evolutionary changes can be explained by an integrated cooperation between organisms, in which symbiosis acts, not as an exception, but as the dominant rule in nature. To put it in a nutshell, is the development of life a saltational symbiotic process or a gradual process, oriented by natural selection and leading organisms to adaptation? In a way, this dilemma may be found in two positions clearly expressed by Theodosius Dobzhansky and Jan Sapp. In 1973, Theodosius Dobzansky wrote his famous article entitled "Nothing in biology makes sense except in the light of evolution", where he transmits the neo-Darwinist view of evolution. Thirty years after this article, Jan Sapp (2003) includes in his book, "Genesis: the Evolution of Biology", a new paradigmatic concept: "Nothing in evolution makes sense except in the light of symbiosis", giving shape to the new ideas on the process of evolution, involving symbiotic principles ${ }^{4}$.

Associated to the new symbiotic approach of biological world there is the concept of organism. This concept is important in the way that the organism must be seen as a symbiotic complex as it was postulated by Jan Sapp": "every eukaryote is a superorganism, a symbiome composed of chromosomal genes, organellar genes, and often other bacterial symbionts as well as viruses. The symbiome, the limit of the multicellular organism, extends beyond the activities of its own cells. All plants and animals involve complex ecological communities of microbes, some of which function as commensals, some as mutualists, and others as parasites, depending on their nature and context". This innovative idea reinforces the approach to a new biology, incorporating the fact that all individuals of a species contain associated bacterial populations that can act in the determination of the phenotype. In this sense, the eukaryotes are not entities genetically unique, and the concept of individual must be seen as a complex biological ecosystem, composed by multiple interdependent parts living symbiotically. It is at the symbiome level, composed by an integrate multigenomic genetic pool, in which natural selection acts.

In this context, we believe that this new biological reality can be tested in phyllogenetical studies of well known taxa, in which molecular data of the individual and of a selected set of its key symbionts should be considered in concert, according to the symbiome concept.

As we have shown in a recent paper ${ }^{10}$, to understand evolution we must extend the tenets used from the Darwinian and neo-Darwinian traditional contribution to explain speciation and adaptation based on competition, to a more broadly and innovative set of concepts including cooperative and synergistic principles, that could be able to build a symbiogenic scenario of evolution (Symbiogenic Theory of Evolution). This theory should be able to include new approaches to the problem of the origin of life, as it was postulated in that work, allowing to have a better and a more real approach to the 
structure and dynamics of the biological world and to understand the life formation on Earth and eventually in other astronomical bodies.

\section{A DYNAMICAL APPROACH FOR EVOLUTION}

Since the 1859 Charles Darwin's book “On the Origin of Species", evolution is considered the core organizing concept and the structural pillar of modern biology. One of the main concepts that Darwin contributed to change radically was the idea of the constancy of species, which allowed the development of the theory of common descent and also challenged the natural theology principles that had ruled natural science for centuries ${ }^{12}$. For the natural theology, the order in nature was the convincing proof of a supreme being, that could explain the harmony and the purpose of the creation. For this reason, we can understand the difficulties and the resistance that these new ideas have when they were introduced to the general society.

As it was well referred by Jan Sapp in his book "Genesis: the evolution of biology"4, evolution is a complementary process of divergence and integration. Divergence in the production of new life forms, and integration when entities join to form new ones. In this context, evolution is a dynamic process that evolves and respond not in the sense of perfection or progress, but in the sense of adaptation to new conditions. We can ask in what way symbiosis can be involved or associated to evolution? In our point of view, symbiosis is the way through which the acquisition of new genomes and new metabolic capacities occur, making possible the evolutive construction of biological organisms. As it was referred by Joshua Lederberg ${ }^{11}$, endosymbiosis is comparable to hibridation, allowing the introduction of genomes philogenetically distincts into associations of organisms with its own characteristics. We believe that it is held to have played a central role in the pre-biotic evolution, in the emergency and evolution of eukaryotes, in the origin of land plants, and in a myriad of adaptive evolutionary innovations ${ }^{4}$. It is at the basis of important ecosystems from deep-sea vents to the most biodiverse communities on earth, such as rainforests and coral reefs ${ }^{4}$. The development of these new evolutive characteristics by the associated organisms is inconsistent with the main tenets of neo-Darwinism, but represents, in our opinion, the main rule in nature, and the main evolutionary mechanism in the establishment and maintenance of biomes, as well as the foundation of biodiversity.

The dynamics of biological processes are mainly characterised, not by the isolation of characteristics from other life forms, but by the integration of those biological capacities into the organisms in evolution. We think that one of the main characteristics of the biological systems is to establish associations or connections and communication with other organisms, which includes this manifestation as one of the main characteristics and diversity of life. In some way, life has not established or developed to exist alone. The complexity of the biological world are rooted to the hierarchical structure of living systems, and all the main issues of biology are problems of hierarchical organization. As Ernst Mayr referred in his brillant book "The Growth of Biological Thought. Diversity Evolution and Inheritance""12, we can deal with two kinds of hierarchies. One is represented by "constitutive hierarchies" and the other the "aggregational hierarchy". In both cases they interfer in the correct understanding of biology, since diversity in the living world exists at every hierarchical level ${ }^{12}$.

In the same book, Mayr refers to the coming of evolutionism in a very clear and elegant way: "Evolution, in a way, contradicts common sense. The offspring of any organism always develops again into the parental type. A cat always produces only cats. To be sure, prior to the acceptance of evolution there have been theories of sudden change. For instance, there was the belief in spontaneous generation, as well as that of heterogony, a belief that seeds of one species of plants, let us say wheat, could occasionally produce plants of a different species, such as rye. But both of these were theories of origins and neither of them had nothing to do with evolution. It required a veritable intellectual revolution before one could even conceive of evolution" 12 . In fact, one of the greatest impediment to understanding evolution in biological world was the fact that this phenomenon can not be observed directly like it occurs in physics or chemistry. Instead, evolution can only be inferred. As it was clearly referred by Sapp" "symbiosis as a source of evolutionary change was in discordance with the central tenets of the evolutionary synthesis of the 1930s and 1940s. That synthesis was bilt on the premise that natural selection acted on small hereditary differences between individuals in interbreeding populations. Although microbial symbionts had been described in many protozoa, worms, sponges, coral, hydra, molluscs, as well as insects and plants, they were either ignored or treated as exceptions, curiosities, special aspects of life. The evolutionary synthesis was essentially a sterile view of evolution without microbes". 


\section{ARE COMPETITION AND COOPERATION ANTAGONICS IN EVOLUTION?}

This question summarises an important issue that has divided the scientific community for more than 150 years. Even, as we believe, that symbiosis is the main rule in the organization of the living world and plays a significative role in heredity and development and beeing considered a general mechanism of evolutionary innovation ${ }^{5}$, we understand that the forces behind the evolutive process are often opposite in the way they act in the theatre of evolution. However, the creative and the novelty effects of symbiosis "were overshadowed by illustrations of conflict and competition in nature, a view of nature which, it had been argued, only reflected views of human social progress. The evolution of cooperation between individuals of different species had been left largely unexplored"5. We believe that competition and cooperation can co-exist in the same scenario of evolution, and probably takes place in terms of discontinuous bursts of activity, depending the internal and external conditions that drives evolution. It means that the same population can evolve using competitive or cooperative processes during the time and space of the evolution scenario.

\section{CONCLUDING REMARKS}

The understanding of the natural world was and is an important goal for mankind. For many scientists, the comprehension of nature is based on the assumption that "nature is competitive, and cooperation is a strange case that needs to be explained" ". In this sense, we have presented several ideas about symbiosis in evolution, involving concepts that are not commly taught or considered in biology. Evolution is usually taught as the result of mutations and genetic recombinations combined with natural selection, but most living forms have symbiotic relationships with microorganisms, and so symbiogenesis seems to play a very important role in the origin and life evolution. Symbiosis is an important support for the acquisition of new genomes and new metabolic capacities which drives living forms evolution. In this sense, the evolutionary changes can be explained by an integrated cooperation between organisms, in which symbiosis acts, not as an exception, but rather than the rule in nature. Beginning with the eukaryotic cell formation, symbiogenesis appears to be the main evolutionary mechanism in the establishment and maintenance of biomes, as well as the foundation of biodiversity, based on rather suddenly evolutionary novelty, which challenges the Darwinian gradualism. These principles can be applied to the life on Earth and beyond.

\section{ACKNOWLEDGEMENTS}

Granted by the Luso-American Foundation (FLAD). The authors are grateful for the technical assistance of Maria Helena Costa and Helena Carrapiço.

\section{REFERENCES}

1. G.V.Levin, "Mars life - how Darwinian pressures might have shaped its form and function", Instruments, Methods, and Missions for Astrobiology, Proceedings of SPIE 5906, 59060D-1 - 59060D-10 (2005).

2. J. Maynard Smith and E. Szathmary, The Origins of Life, Oxford University Press, New York, 1999.

3. L. Margulis and R. Fester (eds.), Symbiosis as a Source of Evolutionary Innovation: Speciation and Morphogenesis. The MIT Press, Cambridge, 1991.

4. J. Sapp, Genesis: The Evolution of Biology, Oxford University Press, New York, 2003.

5. J. Sapp, "The dynamics of symbiosis: an historical overview", Can. J. Bot. 82,1046-1056 (2004).

6. R. Honegger, "Simon Schwendener (1829-1919) and the dual hypothesis of lichens", Bryologist 103, 167-183 (2000).

7. M. Speidel, "The Parasitic Host: Symbiosis contra Neo-Darwinism", Pli 9, 119-138 (2000).

8. A. de Bary, "Ueber Symbiose", Tageblatt 51. Versamml. Deutscher Naturforscher u. Aerzte, Cassel, 121-126 (1878).

9. C. Merezhkowsky, "The Theory of Two Plasms as Foundation of Symbiogenesis. A New Doctrine on the Origins of

Organisms", Proceedings Studies of the Imperial Kazan University 12, 1-102. Publ. Office Imp Univ., Kazan (1909). 
10. F. Carrapiço and T. Rodrigues, "Symbiogenesis and the Early Evolution of Life", Instruments, Methods, and Missions for Astrobiology, Proceedings of SPIE 5906, 59060R-1 - 59060R-4 (2005).

11. J. Lederberg, "Cell Genetics and Hereditary Symbiosis", Physiol. Rev., 32, 403-30 (1952).

12. E. Mayr, The Growth of Biological Thought. Diversity, Evolution and Inheritance, Harvard University Press, Cambridge, 1982. 